\title{
Genetic Polymorphism of Cancer Susceptibility Genes and HPV Infection in Cervical Carcinogenesis
}

\author{
Osamu Nunobiki, ${ }^{1}$ Masatsugu Ueda, ${ }^{2}$ Eisaku Toji, ${ }^{2}$ Michiko Yamamoto, ${ }^{2}$ \\ Kyoko Akashi, ${ }^{3}$ Naomi Sato, ${ }^{2}$ Shinji Izuma, ${ }^{2}$ Kiyo Torii, ${ }^{2}$ Ichiro Tanaka, ${ }^{2}$ \\ Yoshiaki Okamoto, ${ }^{2}$ and Sadamu Noda ${ }^{2}$ \\ ${ }^{1}$ Department of Medical Technology, Kobe Tokiwa University, 6-2 2 chome, Ohtanicho, Nagataku, Hyogo, Kobe 653-0838, Japan \\ ${ }^{2}$ Department of Cytopathology and Gynecology, Osaka Cancer Prevention and Detection Center, Osaka 536-8588, Japan \\ ${ }^{3}$ Department of Obstetrics and Gynecology, Japanese Red Cross Kyoto Daiichi Hospital, Kyoto 605-0981, Japan
}

Correspondence should be addressed to Osamu Nunobiki, o-nunobiki@kobe-tokiwa.ac.jp

Received 8 January 2011; Accepted 3 March 2011

Academic Editor: Kiyomi Taniyama

Copyright (C) 2011 Osamu Nunobiki et al. This is an open access article distributed under the Creative Commons Attribution License, which permits unrestricted use, distribution, and reproduction in any medium, provided the original work is properly cited.

\begin{abstract}
It is widely accepted that specific human papillomavirus (HPV) types are the central etiologic agent of cervical carcinogenesis. However, a number of infected women do not develop invasive lesions, suggesting that other environmental and host factors may play decisive roles in the persistence of HPV infection and further malignant conversion of cervical epithelium. Although many previous reports have focused on HPV and environmental factors, the role of host susceptibility to cervical carcinogenesis is largely unknown. Here, we review the findings of genetic association studies in cervical carcinogenesis with special reference to polymorphisms of glutathione-S-transferase (GST) isoforms, p53 codon 72, murine double-minute 2 homolog (MDM2) gene promoter 309, and FAS gene promoter -670 together with HPV types including our recent research results.
\end{abstract}

\section{Introduction}

Cervical cancer is the second most common cancer in women worldwide, and is both a preventable and a curable disease especially if identified at an early stage. It is widely accepted that specific human papillomavirus (HPV) types are the central etiologic agent of cervical carcinogenesis. Other environmental and host factors also play decisive roles in the persistence of HPV infection and further malignant conversion of cervical epithelium [1]. Although many previous reports have focused on HPV environmental factors, the role of host susceptibility to cervical carcinogenesis is largely unknown.

A large number of previous studies have suggested the possible correlation between genetic polymorphisms of cancer susceptibility genes and the higher risk of human malignant tumors $[2,3]$. Genetic studies lead to a true association are expected to increase understanding of the pathogenesis of each malignancy and to be a powerful tool of prevention and prognosis in the future. Here, we review the findings of genetic polymorphisms of several cancer susceptibility genes together with HPV types in cervical carcinogenesis based on our recent research results using exfoliated cervical cell samples or human cervical squamous carcinoma cell lines. Our studies were approved by our institutional ethics committee, and all samples were obtained with informed consent. To compare the polymorphic features of each genotype and HPV status between normal, LSIL, and HSIL groups, Fisher's exact test or Pearson's chi-square test was used. A level of $P<.05$ was accepted as statistically significant.

\section{Glutathione-S-Transferase GSTM1, GSTT1 Polymorphisms}

The genes of glutathione-S-transferase (GST) family encode enzymes that appear to be critical in cellular protection against the cytotoxic effects. GSTs play an important role in conjugating glutathione to the products of endogenous lipid peroxidation and inactivating organic hydroperoxides via selenium-independent glutathione peroxidase activity, thus 
protecting the cell from the deleterious effects of oxidative stress [4]. GST isoforms GSTM1 and GSTT1 gene deletions may promote the development of cervical dysplasia by moderating the activation and detoxification of polycyclic hydrocarbons and other compounds that influence oxidative stress and DNA adduct formation [5].

We conducted GST genotype analysis together with HPV typing in a total of 198 cervical smear samples obtained from the patients who received cervical cancer screening. They consist of 54 normal, 102 low-grade squamous intraepithelial lesion (LSIL), and 42 high-grade SIL (HSIL). The protocol of this study was approved by our institutional review board, and all samples were obtained from Japanese women with informed consent. The exfoliated cervical cells were disrupted with lysis buffer, and genomic DNA was extracted with phenol-chloroform and precipitated with ethanol using standard techniques. The GSTM1 and GSTT1 genetic polymorphisms were evaluated using multiplex PCR techniques according to the method reported by Chen et al. [6] with some modifications as previously described [2]. The presence of various HPV types was examined by L1-PCR system using published consensus primers (L1C1 and L1C2) [7] according to the method reported by Nagano et al. [8].

Figure 1 shows an example for genotyping of GSTM1 and GSTT1. The polymorphic deletion of the GSTM1 and GSTT1 genes was determined by multiplex PCR. The absence of 215 or $480 \mathrm{bp}$ fragment indicated null GSTM1 or GSTT1 genotype, respectively. Table 1 shows frequency of high-risk HPV and GSTM1, GSTT1 polymorphisms in 198 exfoliated cervical cell samples examined. The 42 patients with HSIL had significantly higher frequency of high-risk HPV than 102 with LSIL and 54 controls. There was no significant difference in the frequency of null GSTM1 genotype between SILs and controls, whereas the 42 patients with HSIL had statistically higher frequency of null GSTT1 genotype than 102 with LSIL and 54 controls. As shown in Table 2, the 31 patients with HSIL had also statistically higher frequency of null GSTT1 genotype than 28 with LSIL among the 69 patients with high-risk HPV.

Previous epidemiological studies of GST and cervical neoplasia found no significant differences in the frequency of GSTM1 or GSTT1 in women with SIL or cancer compared to controls with normal cervical pathology $[5,9,10]$. In our investigation using exfoliated cervical cell samples from a Japanese population, the GSTT1 null genotype was more common among HSIL cases than LSIL cases and controls. Moreover, the patients with HSIL also had higher frequency of null GSTT1 genotype than those with LSIL among highrisk HPV group. GSTT1 differs from other classes of GSTs in its lack of activity towards the GST model substrate 1-chloro-2,4-dinitrobenzene and its failure to bind to Shexyl-glutathione affinity matrices [11]. The gene defect of GSTT1 was reported to be associated with an increased risk of myelodysplastic syndromes [12], astrocytoma, and meningioma [13]. We previously examined GSTM1 and GSTT1 genotypes in 104 cell lines originating from a variety of human malignant tumors and found that GSTT1 null genotype was more common in cervical cancer cells [2]. It might be of interest to further examine the difference

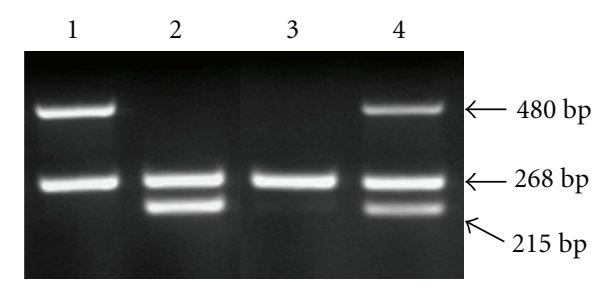

Figure 1: Genotyping of GSTM1 and GSTT1 by multiplex PCR. Lane 1: null GSTM1 genotype (absence of $215 \mathrm{bp}$ fragment). Lane 2: null GSTT1 genotype (absence of $480 \mathrm{bp}$ fragment). Lane 3: null GSTM1 and GSTT1 genotypes (absence of 215 and $480 \mathrm{bp}$ fragments). Lane 4: present GSTM1 and GSTT1 genotypes. $\beta$ globin as a positive control is detected as $268 \mathrm{bp}$ fragment.

TABle 1: Frequency of high-risk HPV and GSTM1, GSTT1 polymorphisms in exfoliated cervical cell samples.

\begin{tabular}{lccc}
\hline Lesions & $\begin{array}{c}\text { Number with } \\
\text { high-risk HPV }\end{array}$ & GSTM1null & GSTT1 null \\
\hline Normal $(n=54)$ & $10(18.5 \%)^{\mathrm{a}}$ & $28(51.9 \%)$ & $24(44.4 \%)^{\mathrm{c}}$ \\
LSIL $(n=102)$ & $28(27.5 \%)^{\mathrm{b}}$ & $55(53.9 \%)$ & $40(39.2 \%)^{\mathrm{d}}$ \\
HSIL $(n=42)$ & $31(73.8 \%)^{\mathrm{a}, \mathrm{b}}$ & $20(47.6 \%)$ & $\begin{array}{c}29 \\
(69.0 \%)^{\mathrm{c}, \mathrm{d}}\end{array}$ \\
\hline
\end{tabular}

${ }^{\mathrm{a}} P<.0001, \mathrm{OR}=12.4 \chi^{2}$ versus normal. ${ }^{\mathrm{b}} P<.0001, \mathrm{OR}=7.4 \chi^{2}$ versus LSIL. ${ }^{\mathrm{c}} P=.0162, \mathrm{OR}=2.8 \chi^{2}$ versus normal. ${ }^{\mathrm{d}} P=.0011$, OR $=3.5$ $\chi^{2}$ versus LSIL.

TABLE 2: HPV status and frequency of GSTT1 polymorphism in exfoliated cervical cell samples.

\begin{tabular}{lcc}
\hline Study group & $n$ & GSTT1null \\
\hline High-risk HPV- & & \\
$\quad$ Normal & 44 & $20(45.5 \%)$ \\
LSIL & 74 & $31(41.9 \%)$ \\
HSIL & 11 & $8(72.7 \%)$ \\
\hline High-risk HPV+ & & $4(40.0 \%)$ \\
$\quad$ Normal & 10 & $9(32.1 \%)^{\mathrm{a}}$ \\
LSIL & 28 & $21(67.7 \%)^{\mathrm{a}}$ \\
$\quad$ HSIL & 31 & \\
${ }^{\mathrm{a}} P=.0063$, OR $=4.4 \chi^{2}$ versus LSIL. &
\end{tabular}

in the polymorphic frequency of the null GSTT1 genotype between SILs and invasive cervical cancer to clarify whether this genotype alteration occurs prior to the development of malignant phenotype cells or late in the development of neoplastic cells.

\section{3. p53 Codon 72 Polymorphism}

p53 is a tumor suppressor gene involved in multiple pathways including apoptosis, cellular transcriptional control, and cell cycle regulation $[14,15]$. A large number of human tumors, including smoke-induced lung cancer, show mutations and deletions of the p53 gene that result in loss of tumor suppression function and cell cycle deregulation [16]. A polymorphism at codon 72 of the p53 gene results in the substitution of arginine (Arg) for proline (Pro) in the gene 


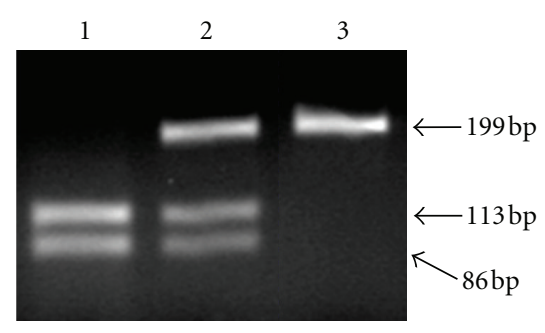

Figure 2: Genotyping of p53 codon 72 by PCR-RFLP. Lane 1: Arg/Arg homozygote. Lane 2: Arg/Pro heterozygote. Lane 3: Pro/Pro homozygote. The fragment of $199 \mathrm{bp}$ is the nondigested PCR product from the Pro allele. Fragments of 113 and $86 \mathrm{bp}$ result from BstUI digestion of the Arg allele.

TABLE 3: Frequency of high-risk HPV and p53 codon 72 polymorphism in exfoliated cervical cell samples.

\begin{tabular}{lcccc}
\hline Lesions & Number with & \multicolumn{3}{c}{ Aminoacid at p53 codon 72 } \\
& high-risk HPV & Arg & Arg/Pro & Pro \\
\hline $\begin{array}{l}\text { Normal } \\
(n=54)\end{array}$ & $10(18.5 \%)^{\mathrm{a}}$ & $24(44.4 \%)$ & $23(42.6 \%)$ & $7(13.0 \%)$ \\
$\begin{array}{l}\text { LSIL } \\
(n=102)\end{array}$ & $28(27.5 \%)^{\mathrm{b}}$ & $38(37.3 \%)$ & $40(39.2 \%)$ & $24(23.5 \%)$ \\
$\begin{array}{l}\text { HSIL } \\
(n=42)\end{array}$ & $31(73.8 \%)^{\mathrm{a}, \mathrm{b}}$ & $18(42.9 \%)$ & $16(38.1 \%)$ & $8(19.0 \%)$ \\
$\begin{array}{l}{ }^{\mathrm{a}} P<.0001, \text { OR }=12.4 \chi^{2} \text { versus normal. }{ }^{\mathrm{b}} P<.0001, \text { OR }=7.4 \chi^{2} \text { versus } \\
\text { LSIL. }\end{array}$
\end{tabular}

product. It has been suggested that the homozygous Arg genotype increased the susceptibility of $\mathrm{p} 53$ protein to degradation by E6 protein derived from oncogenic HPV [17].

We conducted genotype analysis of p53 codon 72 together with HPV typing in a total of 198 cervical smear samples obtained from the patients who received cervical cancer screening. They consist of 54 normal, 102 LSIL, and $42 \mathrm{HSIL}$, as described above. PCR restriction fragment length polymorphism (RFLP) analysis of codon 72 of the p53 gene, which was modified from a technique described by Ara et al. [18], was conducted to identify p53 genotypes. HPV types were examined using L1-PCR, as describer above.

As shown in Figure 2, the Arg allele is cleaved by BstUI and yields two small fragments (113 and $86 \mathrm{bp}$ ). The Pro allele is not cleaved by BstUI and has a single $199 \mathrm{bp}$ band. The heterozygote contains three bands (199, 113, and $86 \mathrm{bp}$ ). Table 3 shows HPV status and polymorphic frequency of p53 codon 72 in 198 samples examined. The 42 patients with HSIL had significantly higher frequency of high-risk HPV than 102 with LSIL and 54 controls, as described above. The differences in the polymorphic frequency of p53 Arg, Arg/Pro, and Pro genotypes between SILs and controls were statistically not significant. When the Arg genotype was compared to the Arg/Pro + Pro genotypes, there was again no statistical difference in the genotype prevalence between SILs and controls with or without high-risk HPV, as shown in Table 4.

Our present results revealed that the differences in the polymorphic frequency of p53 Arg, Arg/Pro, and Pro
TABLE 4: HPV status and frequency of $\mathrm{p} 53$ codon 72 polymorphism in exfoliated cervical cell samples.

\begin{tabular}{lccc}
\hline Study group & $n$ & \multicolumn{2}{c}{ Aminoacid at p53 codon 72} \\
& & Arg & Arg/Pro + Pro \\
\hline $\begin{array}{l}\text { High-risk HPV- } \\
\quad \text { Normal }\end{array}$ & 44 & $20(45.5 \%)$ & $24(54.5 \%)$ \\
LSIL & 74 & $26(35.1 \%)$ & $48(64.9 \%)$ \\
$\quad$ HSIL & 11 & $4(36.4 \%)$ & $7(63.6 \%)$ \\
\hline High-risk HPV+ & & & \\
$\quad$ Normal & 10 & $4(40.0 \%)$ & $6(60.0 \%)$ \\
LSIL & 28 & $12(42.9 \%)$ & $16(57.1 \%)$ \\
HSIL & 31 & $14(45.2 \%)$ & $17(54.8 \%)$ \\
\hline
\end{tabular}

genotypes between SILs and controls were statistically not significant. Moreover, neither Arg nor Pro allele affected the increased risk of SILs with or without high-risk HPVs compared to controls. Some previous studies have reported no correlation between germline polymorphisms of the p53 codon 72 and increased risk of cervical cancer [19-21]. The other study reported by Nishikawa et al. [22] using cervical condyloma, dysplasia, and cancer tissue samples demonstrated that no statistically significant differences in the distribution of $\mathrm{p} 53$ genotypes were found among the patients with these diseases, regardless of HPV status. The recent meta-analysis on p53 codon 72 polymorphism reported by Klug et al. [23] also demonstrated that no statistically significant differences in the distribution of p53 genotypes were found among the patients with cervical diseases. These data suggest that the p53 codon 72 polymorphism is unlikely to be associated with the development of HPV-associated cervical neoplasms.

\section{MDM2-SNP309}

Murine double-minute 2 homolog (MDM2) is the key negative regulator of $\mathrm{p} 53$, and dysfunction of these genes may be associated with an increased rate of accumulation of genetic errors, thereby enhancing the progression of the disease. A single nucleotide polymorphism (SNP) in the MDM2 gene promoter, SNP309 (a T to G change at nucleotide 309 in the first intron), increases the affinity of the promoter for the transcription activator Sp1, resulting in higher level of MDM2 mRNA and MDM2 protein and a subsequent attenuation of the p53 pathway [24]. SNP309 occurs at a relatively high frequency in the general population and has been shown to be associated with accelerated tumorigenesis and the timing of cancer onset [24-27]. However, there have been very few reports on the correlation between SNP of MDM2 gene and cervical cancer susceptibility [28].

We conducted genotype analysis of MDM2-SNP309 together with HPV typing in a total of 195 cervical smear samples obtained from patients with consent who received cervical cancer screening. They consist of 52 normal, 102 LSIL, and 41 HSIL. Eight human cervical squamous carcinoma cell lines (SKG-I, SKG-II, SKG-IIIa, SKG-IIIb, OMC-1, YUMOTO, QG-U, and QG-H) were also used 
TABLE 5: Frequency of high-risk HPV and MDM2-SNP309 in exfoliated cervical cell samples.

\begin{tabular}{|c|c|c|c|c|c|}
\hline \multirow{2}{*}{ Lesions } & \multirow{2}{*}{ Number with high-risk HPV } & \multicolumn{2}{|c|}{ Genotype frequency } & \multicolumn{2}{|c|}{ Allele frequency } \\
\hline & & $\mathrm{TT}$ & $\mathrm{TG}+\mathrm{GG}$ & $\mathrm{T}$ & G \\
\hline Normal $(n=52)$ & $10(19.2 \%)^{\mathrm{a}}$ & $11(21.2 \%)$ & $41(78.8 \%)$ & $49(47.2 \%)$ & $55(52.8 \%)$ \\
\hline LSIL $(n=102)$ & $28(27.5 \%)^{\mathrm{b}}$ & $26(25.4 \%)$ & $76(74.6 \%)$ & $104(50.6 \%)$ & $100(49.4 \%)$ \\
\hline $\operatorname{HSIL}(n=41)$ & $30(73.2 \%)^{\mathrm{a}, \mathrm{b}}$ & $7(17.1 \%)$ & $34(82.9 \%)$ & $37(45.1 \%)$ & $45(54.9 \%)$ \\
\hline
\end{tabular}

${ }^{\mathrm{a}} P=.0010 \chi^{2}$ versus normal. ${ }^{\mathrm{b}} P=.0019 \chi^{2}$ versus LSIL.

TABLE 6: HPV status and frequency of MDM2-SNP309 in exfoliated cervical cell samples.

\begin{tabular}{|c|c|c|c|c|c|c|}
\hline \multirow{2}{*}{ Study group } & \multirow{2}{*}{$n$} & \multicolumn{2}{|c|}{ Genotype at MDM2-SNP309 } & \multirow{2}{*}{ OR } & \multirow{2}{*}{$95 \%$ CI } & \multirow{2}{*}{$P$ value } \\
\hline & & TT & $\mathrm{TG}+\mathrm{GG}$ & & & \\
\hline \multicolumn{7}{|c|}{ High-risk HPV- } \\
\hline Normal & 42 & $8(18.2 \%)$ & $36(81.8 \%)$ & 1 & & \\
\hline LSIL & 74 & $14(18.4 \%)$ & $60(81.1 \%)$ & 0.95 & $0.36-2.49$ & .921 \\
\hline HSIL & 11 & $4(36.7 \%)$ & $7(63.3 \%)$ & 0.41 & $0.10-1.59$ & .186 \\
\hline \multicolumn{7}{|c|}{ High-risk HPV+ } \\
\hline Normal & 10 & $3(30.0 \%)$ & $7(70.0 \%)$ & 1 & & \\
\hline LSIL & 28 & $12(42.9 \%)$ & $16(57.1 \%)$ & 0.38 & $0.10-1.42$ & .475 \\
\hline HSIL & 30 & $3(10.0 \%)$ & $27(90.0 \%)$ & 8.88 & $2.34-33.63$ & .003 \\
\hline
\end{tabular}

OR: odds ratio.

CI: confidence interval.

for genotype analysis of MDM2-SNP309 together with HPV typing. All cell lines were originating from Japanese women, as previously described [2]. For MDM2-SNP309 genotyping, two independent PCR assays for each allele, modified from a technique described by Menin et al. [25], were performed using primer pairs specific for the two alleles. HPV types were examined using L1-PCR, as describer above.

Figure 3 shows a representative genotyping of MDMSNP309 by two independent PCR assays. The wild-type (T) and the mutant $(\mathrm{G})$ allele yield 121-bp and 168-bp fragment, respectively. Table 5 shows the frequency of highrisk HPV and MDM2-SNP309 in 195 exfoliated cervical cell samples examined. When TT genotype was compared to TG + GG genotype, 41 patients with HSIL had significantly higher frequency of high-risk HPV than 102 with LSIL and 52 controls; however, there were no statistical significant differences in the TG + GG genotype prevalence and allele frequencies between SILs and controls. No statistical difference was also found in the genotype frequency of MDM2-SNP 309 between SILs and controls among 127 patients without high-risk HPV. However, there was an increased OR for TG + GG genotype in HSIL cases compared to controls among 68 patients with high-risk HPV, as shown in Table 6. Interestingly, 21 cases with HPV types 16 and/or 18 had significantly higher frequency of the TG + GG genotype and $\mathrm{G}$ allele than 47 with other types of high-risk $\mathrm{HPV}$, as indicated in Table 7. Moreover, as shown in Figure 4, genotyping of MDM2-SNP309 in 8 cervical squamous carcinoma cell lines revealed that TT genotype was detected only in the SKG-IIIa cell line, whereas the other 7 of 8 (87.5 $\%$ ) cell lines had TG or GG genotype. In addition, 7 of 8 cell lines except for YUMOTO were positive for high-risk HPV.

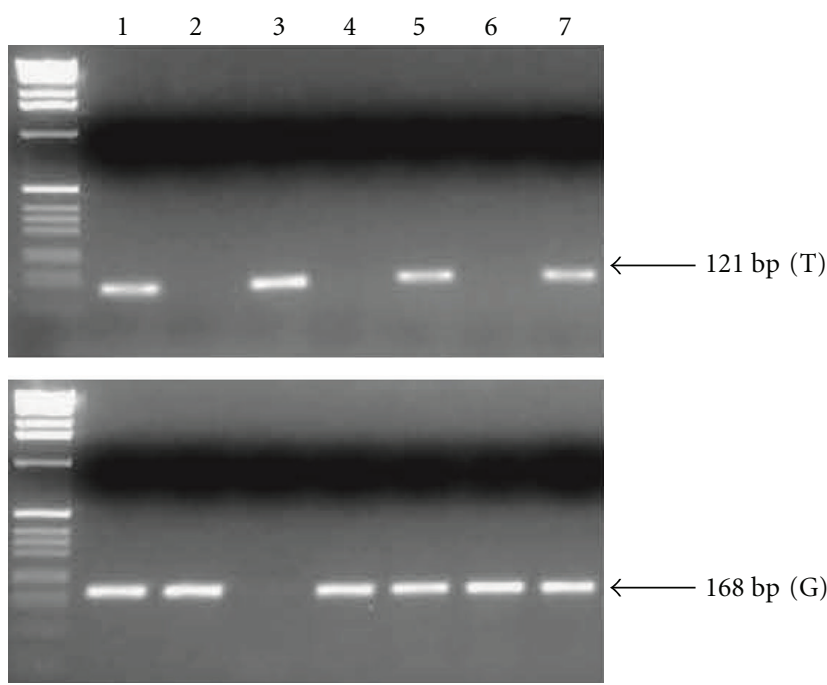

FIGURE 3: Representative genotyping of MDM2-SNP309 by two independent PCR assays for each allele. Lanes 1, 5, and 7: TG heterozygote. Lanes 2, 4, and 6: GG homozygote. Lane 3: TT homozygote. The wild-type $(\mathrm{T})$ and the mutant $(\mathrm{G})$ allele yields 121-bp and 168-bp fragments, respectively.

Recently, Meissner Rde et al. [28] tested the hypothesis that this functional variant in the MDM2 promoter was associated with either risk or early age diagnosis of cervical cancer in a Brazilian population. A primer-introduced restriction analysis PCR assay was used to genotype the MDM2-SNP309 of 72 cervical carcinoma patients and 100 healthy women. However, no statistically significant association was observed between SNP309 and cervical cancer. Moreover, they could not find allele or genotype frequency differences between 
TABLE 7: High-risk HPV types and frequency of MDM2-SNP309 in exfoliated cervical cell.

\begin{tabular}{lcccc}
\hline \multirow{2}{*}{ Study group } & \multicolumn{2}{c}{ Genotype frequency } & \multicolumn{2}{c}{ Allele frequency } \\
& TT & TG + & T & G \\
\hline HPV types & & & & \\
16,18 & $2(9.5 \%)$ & $19(90.5 \%)$ & $14(33.3 \%)$ & $28(66.7 \%)^{\mathrm{b}}$ \\
$(n=21)$ & & & & \\
HPV other & & & & \\
types & $18(38.2 \%)$ & $29(61.8 \%)^{\mathrm{a}}$ & $51(54.2 \%)$ & $43(45.8 \%)^{\mathrm{b}}$ \\
$(n=47)$ & & & & \\
\hline${ }^{\mathrm{a}} P=.0161 \chi^{2}$ versus HPV types $16,18$. \\
${ }^{\mathrm{b} P=.0240} \chi^{2}$ versus HPV types $16,18$.
\end{tabular}

the group of patients with cancer diagnosis at an early age (younger than 40 years old) and the group of older patients. In contrast, Arvanitis and Spandidos [29] demonstrated that MDM2 was one of the potential candidates for the development of cervical neoplasms. They analyzed the mRNA expression profiles of $24 \mathrm{G} 1 / \mathrm{S}$ checkpoint genes in cancer and SIL of the uterine cervix. In total 35 squamous cervical carcinomas, 26 HSIL, 33 LSIL tissues, and 28 normal uterine cervix specimens as controls were assessed by RTPCR. MDM2 was found to be upregulated in SIL, while RBL1 was found to be downregulated in all three groups of cases.

Our present results using exfoliated cervical cell samples demonstrated that there was an increased OR for TG + GG genotype in HSIL cases compared to controls among the patients with high-risk HPV. We observed that HPV types 16 and 18 , the most prevalent and aggressive types worldwide, are predominant in cases with TG + GG genotype and G allele. Moreover, 7 of 8 human cervical squamous carcinoma cell lines that possess high-risk HPV except YUMOTO also showed TG or GG genotype. These observations suggest that MDM2-SNP309 and high-risk HPV infection may be cooperatively associated with cervical carcinogenesis. It would be of interest to further evaluate whether MDM2SNP309 has the potential to be used in conjunction with HPV-DNA testing and cervical cytology for the management of SIL patients.

\section{Fas Gene Promoter -670 Polymorphism}

Apoptosis is a physiological process that regulates normal homeostasis, and alterations of apoptosis-related genes are likely to contribute to the pathogenesis of autoimmune diseases [30] and malignant tumors [31]. Among various cell surface death receptors, Fas/CD95, a transmembrane receptor, is known as a member of tumor necrosis factor (TNF) receptors superfamily [32]. Downregulation of Fas with resultant resistance to death signals has been reported in many cancers [33-35]. The transcriptional expression of Fas gene is regulated by a number of genetic elements located in the $5^{\prime}$ upstream promoter region of the gene. SNP at -670 in the enhancer region $(\mathrm{A} / \mathrm{G})$ situates at a binding element of gamma interferon activation signal (GAS). Homozygous for $G$ allele could result in a complete deletion of the binding sequence of transcription element GAS, which is responsible

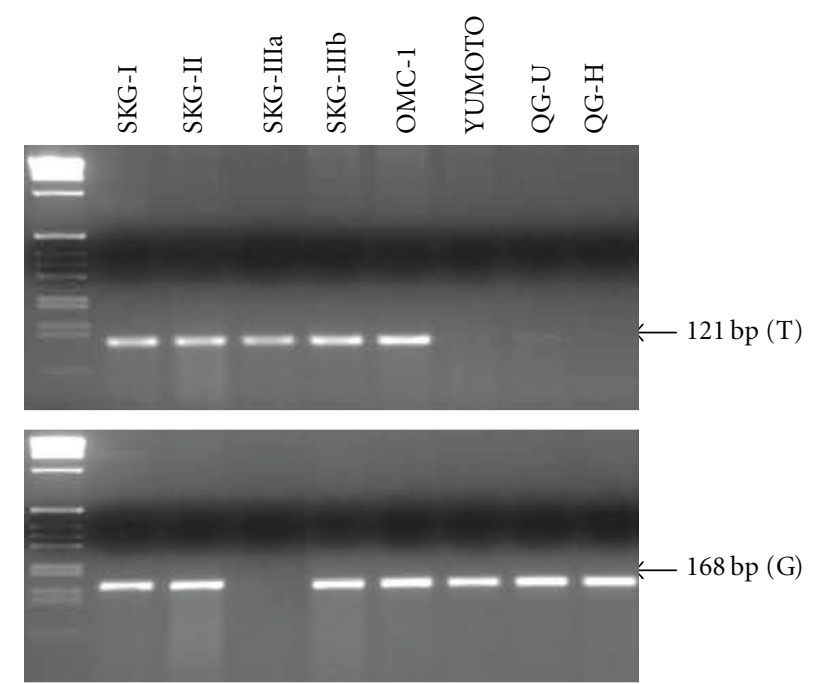

FIGURE 4: Genotyping of MDM2-SNP309 in 8 cervical squamous carcinoma cell lines by two independent PCR assays for each allele. The TT genotype was detected only for SKG-IIIa, whereas the TG genotype for SKG-I, SKG-II, SKG-IIIb, and OMC-1, and the GG genotype for YUMOTO, QG-U, and QG-H cell lines, respectively.

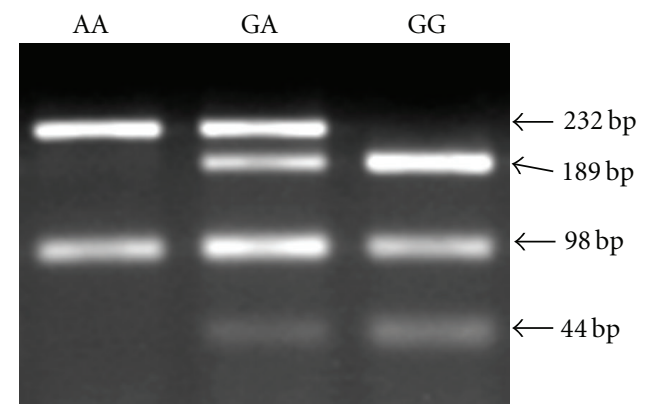

FIGURE 5: Genotyping of Fas gene promoter -670 in DNA samples from peripheral blood lymphocytes by PCR-RFLP. The genotypes AA (232 bp), GA (189, 233 bp), and GG (189bp) are shown.

for the signal emanated through STAT1, and in a significant alteration in the gene expression [36, 37]. However, the correlation between this SNP and cancer susceptibility including the risk of gynecological malignancies has not been extensively studied.

We conducted genotype analysis of Fas gene promoter -670 together with HPV typing in a total of 279 cervical smear samples obtained from the patients with consent who received cervical cancer screening. They consist of 63 normal, 167 LSIL, and 49 HSIL. Eight human cervical squamous carcinoma cell lines (SKG-I, SKG-II, SKG-IIIa, SKG-IIIb, OMC-1, YUMOTO, QG-U, and QG-H) were also used for genotype analysis of this SNP together with HPV typing, as described above. PCR-RFLP analysis of the Fas gene promoter -670 , modified from a technique described by Lee et al. [38], was conducted, and HPV types were examined using L1-PCR, as describer above.

Figure 5 shows an example for genotyping of Fas gene promoter -670 in exfoliated cervical cell samples. The fragments of 232 and $188 \mathrm{bps}$ indicated the AA and GG 
TABLE 8: Frequency of high-risk HPV and Fas promoter -670 polymorphism in exfoliated cervical cell samples.

\begin{tabular}{|c|c|c|c|c|c|}
\hline \multirow{2}{*}{ Lesions } & \multirow{2}{*}{ Number with high-risk HPV } & \multicolumn{2}{|c|}{ Genotype frequency } & \multicolumn{2}{|c|}{ Allele frequency } \\
\hline & & $\mathrm{AA}$ & $\mathrm{GA}+\mathrm{GG}$ & A & $\mathrm{G}$ \\
\hline Normal $(n=63)$ & $10(15.9 \%)^{\mathrm{a}}$ & $19(30.2 \%)$ & $44(69.8 \%)^{\mathrm{c}}$ & $67(53.2 \%)$ & $59(46.8 \%)^{\mathrm{e}}$ \\
\hline LSIL $(n=167)$ & $46(27.5 \%)^{\mathrm{b}}$ & $51(30.5 \%)$ & $116(69.5 \%)^{\mathrm{d}}$ & $165(49.4 \%)$ & $169(50.6 \%)^{\mathrm{f}}$ \\
\hline HSIL & $40(81.6 \%)^{\mathrm{a}, \mathrm{b}}$ & $5(10.2 \%)$ & $44(89.8 \%)^{\mathrm{c,d}}$ & $37(37.8 \%)$ & $61(62.2 \%)^{\mathrm{e}, \mathrm{f}}$ \\
\hline
\end{tabular}

${ }^{\mathrm{a}} P<.0001 \chi^{2}$ versus normal, ${ }^{\mathrm{b}} P<.0001 \chi^{2}$ versus LSIL,

${ }^{\mathrm{c}} P=.0107 \chi^{2}$ versus normal, ${ }^{\mathrm{d}} P=.0043 \chi^{2}$ versus LSIL,

${ }^{\mathrm{e}} P=.0217 \chi^{2}$ versus normal, ${ }^{\mathrm{f}} P=.0422 \chi^{2}$ versus LSIL.

TABLE 9: HPV status and frequency of Fas promoter -670 polymorphism in exfoliated cervical cell samples.

\begin{tabular}{|c|c|c|c|c|c|c|}
\hline \multirow{2}{*}{ Study group } & \multirow{2}{*}{$n$} & \multicolumn{2}{|c|}{ Genotype at Fas promoter -670} & \multirow{2}{*}{ OR } & \multirow{2}{*}{$95 \% \mathrm{CI}$} & \multirow{2}{*}{$P f$} \\
\hline & & $\mathrm{AA}$ & $\mathrm{GA}+\mathrm{GG}$ & & & \\
\hline \multicolumn{7}{|c|}{ High-risk HPV- } \\
\hline Normal & 53 & $15(28.3 \%)$ & $38(71.7 \%)$ & 1 & & \\
\hline LSIL & 121 & $36(29.8 \%)$ & $85(70.2 \%)$ & 0.93 & $0.44-1.95$ & .847 \\
\hline HSIL & 9 & $1(11.1 \%)$ & $8(88.9 \%)$ & 3.16 & $0.40-25.04$ & .276 \\
\hline \multicolumn{7}{|c|}{ High-risk HPV+ } \\
\hline Normal & 10 & $4(40.0 \%)$ & $6(60.0 \%)$ & 1 & & \\
\hline LSIL & 46 & $15(32.6 \%)$ & $31(67.4 \%)$ & 1.38 & $0.34-5.66$ & .655 \\
\hline HSIL & 40 & $4(10.0 \%)$ & $36(90.0 \%)$ & 6.00 & $1.32-27.37$ & .021 \\
\hline
\end{tabular}

OR: odds ratio.

CI: confidence interval.

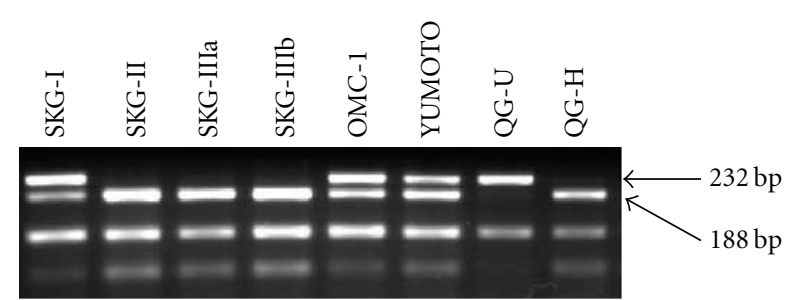

Figure 6: Genotyping of Fas gene promoter -670 in 8 cervical squamous carcinoma cell lines by PCR-RFLP. The AA genotype was detected only for QG-U, whereas the GA genotype for SKGI, OMC-1, and YUMOTO, and the GG genotype for SKG-II, SKGIIIa, SKG-IIIb, and QG-H cell lines, respectively.

genotypes, respectively. The GA genotype contained these two bands. Table 8 shows the frequency of high-risk HPV and Fas promoter -670 polymorphism in 279 samples examined. When AA genotype was compared to GA + GG genotype, 49 patients with HSIL had significantly higher frequency of high-risk HPV and GA + GG genotype than 167 with LSIL and 63 controls. G allele frequency was also higher in HSIL than in LSIL and controls. There was no statistical difference in the GA + GG genotype prevalence between SILs and controls among 183 patients without high-risk HPV as shown in Table 9. However, there was an increased odds ratio (OR) for GA + GG genotype in HSIL cases compared to controls among 96 patients with high-risk HPV. As shown in Figure 6, genotyping of Fas gene promoter -670 in 8 cervical squamous carcinoma cell lines revealed that AA genotype was detected only in the QG-U cell line, whereas the other 7 of $8(87.5 \%)$ cell lines had GA or GG genotype.
Polymorphisms in the promoter region or $5^{\prime}$ flanking region of genes can lead to different levels of gene expression and have been also implicated in a number of diseases. Recently, Lai et al. [39] conducted Fas promoter -670 polymorphism analysis using surgical and biopsy tissue specimens of cervical neoplasm and reported that the frequency of A allele and AA genotype increased in accordance with the multistep carcinogenesis from LSIL, HSIL to invasive squamous cell cancer. They stated that A allele and AA genotype, conferring an intact GAS element and more efficient Fas expression, could be one of the mechanism that cells use to avoid carcinogenesis. In contrast, our present results using exfoliated cervical cell samples demonstrated that the frequency of GA + GG genotype or G allele increased from LSIL to HSIL. Moreover, there was an increased OR for GA + GG genotype in HSIL cases compared to controls among the patients with high-risk HPV. Recently, Engelmark et al. [40] and Dybikowska et al. [41] have demonstrated that AA genotype in Fas gene promoter at -670 position may not be engaged in the development of cervical neoplasia in Swedish and Polish population, respectively. These discrepancies may be due to the ethnic variation of genotype frequency of Fas gene promoter in different geographical regions.

Previous studies $[42,43]$ have demonstrated that highrisk HPV infection is inversely correlated with apoptosis of cervical epithelial cells and that a decrease of apoptosis is closely associated with higher histologic grade of SIL. In cervical cancer tissues and cell lines, significant decrease in the expression levels of Fas has been also reported $[43,44]$. The higher frequency of GA or GG genotype in HSIL cases in our series may result in a significant decrease in Fas 
gene expression and subsequent escape from apoptosis of the cells in high-risk HPV-related cervical carcinogenesis. Interestingly, 7 of 8 human cervical squamous carcinoma cell lines that possess high-risk HPV except for YUMOTO also showed GA or GG genotype. Fas gene promoter polymorphism may be closely associated with cervical carcinogenesis particularly in high-risk HPV group. These observations are potentially important in managing SIL patients by cytologic examination and in understanding the pathogenesis of cervical cancer.

\section{Conclusion and Future Directions}

Here, we review the findings of genetic association studies in cervical carcinogenesis with special reference to polymorphisms of GST isoforms, p53 codon 72, MDM2-SNP309, and FAS gene promoter -670 together with HPV types including our recent research results. Our studies using exfoliated cervical cell samples or human cervical squamous carcinoma cell lines have demonstrated that the GSTT1 null genotype, the TG/GG genotype of MDM2-SNP309, and the GA/GG genotype or G allele of Fas promoter -670 are closely associated with cervical carcinogenesis together with high-risk HPV infection. It would be of interest to further evaluate whether these polymorphisms could be used as a disease marker for the natural history of cervical neoplasms in a setting of longitudinal cohort study and for the determination of appropriate screening interval in patients with or without high-risk HPV.

HPV are the etiologic agents of cervical and other epithelial cancers. Persistence of infections by high-risk HPV types is the single greatest risk factor for malignant progression. Vaccination against HPV types 16 and 18 has commenced or will soon commence in a number of countries. Our studies have demonstrated that the frequency of $\mathrm{G}$ allele increased from LSIL to HSIL and that there was an increased OR for G allele in HSIL cases with high-risk HPV types including 52 and 58. It is known that geographically different oncogenic HPV types 52 and 58 are more prevalent than 16 and 18 in East Asia. HPV prevalence should be considered and treated individually regarding the strategy that best suits the HPV types in a given geographical area. It is likely that novel strategies in combination with vaccination against HPV types 16 and 18 are required in those countries where other types of HPV may be more prevalent. Moreover, further studies on the differential gene expression profiles between normal cervical keratinocytes and cervical cancer cell lines may provide the better understanding for the effect of these polymorphisms in the sequence of cervical carcinogenesis.

\section{References}

[1] H. zur Hausen, "Papillomaviruses causing cancer: evasion from host-cell control in early events in carcinogenesis," Journal of the National Cancer Institute, vol. 92, no. 9, pp. 690698, 2000.

[2] M. Ueda, Y. C. Hung, Y. Terai et al., "Glutathione S-transferase GSTM1, GSTT1 and p53 codon 72 polymorphisms in human tumor cells," Human cell, vol. 16, no. 4, pp. 241-251, 2003.
[3] C. Tsigris, A. Chatzitheofylaktou, C. Xiromeritis, N. Nikiteas, and A. Yannopoulos, "Genetic association studies in digestive system malignancies," Anticancer Research, vol. 27, no. 5B, pp. 3577-3587, 2007.

[4] T. R. Rebbeck, "Molecular epidemiology of the human glutathione S-transferase genotypes GSTM1 and GSTT1 in cancer susceptibility," Cancer Epidemiology Biomarkers and Prevention, vol. 6, no. 9, pp. 733-743, 1997.

[5] M. T. Goodman, K. McDuffie, B. Hernandez et al., "CYP1A1, GSTM1, and GSTT1 polymorphisms and the risk of cervical squamous intraepithelial lesions in a multiethnic population," Gynecologic Oncology, vol. 81, no. 2, pp. 263-269, 2001.

[6] C.-L. Chen, Q. Liu, and M. V. Relling, "Simultaneous characterization of glutathione S-transferase M1 and T1 polymorphisms by polymerase chain reaction in American whites and blacks," Pharmacogenetics, vol. 6, no. 2, pp. 187-191, 1996.

[7] H. Yoshikawa, T. Kawana, K. Kitagawa, M. Mizuno, H. Yoshikura, and A. Iwamoto, "Detection and typing of multiple general human papillomaviruses by DNA amplification with consensus primers," Japanese Journal of Cancer Research, vol. 82, no. 5, pp. 524-531, 1991.

[8] H. Nagano, H. Yoshikawa, T. Kawana et al., "Association of multiple human papillomavirus types with vulvar neoplasias," Journal of Obstetrics and Gynaecology Research, vol. 22, no. 1, pp. 1-8, 1996.

[9] A. Warwick, P. Sarhanis, C. Redman et al., "Theta class glutathione S-transferase GSTT1 genotypes and susceptibility to cervical neoplasia: interactions with GSTM1, CYP2D6 and smoking," Carcinogenesis, vol. 15, no. 12, pp. 2841-2845, 1994.

[10] C. Chen, M. M. Madeleine, N. S. Weiss, and J. R. Daling, "Glutathione S-transferase M1 genotypes and the risk of squamous carcinoma of the cervix: a population-based case-control study," American Journal of Epidemiology, vol. 150, no. 6, pp. 568-572, 1999.

[11] D. J. Meyer, L. G. Christodoulides, K. Hong Tan, and B. Ketterer, "Isolation, properties and tissue distribution of rat glutathione transferase E," FEBS Letters, vol. 173, no. 2, pp. 327-330, 1984.

[12] H. Chen, D. P. Sandler, J. A. Taylor et al., "Increased risk for myelodysplastic syndromes in individuals with glutathione transferase theta 1 (GSTT1) gene defect," Lancet, vol. 347, no. 8997, pp. 295-297, 1996.

[13] J. Elexpuru-Camiruaga, N. Buxton, V. Kandula et al., "Susceptibility to astrocytoma and meningioma: influence of allelism at glutathione S-transferase (GSTT1 and GSTM1) and cytochrome P-450 (CYP2D6) loci," Cancer Research, vol. 55, no. 19, pp. 4237-4239, 1995.

[14] V. Dulic, W. K. Kaufmann, S. J. Wilson et al., "p53-dependent inhibition of cyclin-dependent kinase activities in human fibroblasts during radiation-induced G1 arrest," Cell, vol. 76, no. 6, pp. 1013-1023, 1994.

[15] D. B. Woods and K. H. Vousden, "Regulation of p53 function," Experimental Cell Research, vol. 264, no. 1, pp. 56-66, 2001.

[16] M. S. Greenblatt, W. P. Bennett, M. Hollstein, and C. C. Harris, "Mutations in the p53 tumor suppressor gene: clues to cancer etiology and molecular pathogenesis," Cancer Research, vol. 54, no. 18, pp. 4855-4878, 1994.

[17] A. Storey, M. Thomas, A. Kalita et al., "Role of a p53 polymorphism in the development of human papillomavirusassociated cancer," Nature, vol. 393, no. 6682, pp. 229-234, 1998.

[18] S. Ara, P. S. Y. Lee, M. F. Hansen, and H. Saya, "Codon 72 polymorphism of the TP53 gene," Nucleic Acids Research, vol. 18 , no. 16, p. $4961,1990$. 
[19] A. N. Rosenthal, A. Ryan, R. M. Al-Jehani, A. Storey, C. A. Harwood, and I. J. Jacobs, "p53 codon 72 polymorphism and risk of cervical cancer in UK," Lancet, vol. 352, no. 9131, pp. 871-872, 1998.

[20] S. Lanham, I. Campbell, P. Watt, and R. Gornall, "p53 polymorphism and risk of cervical cancer," Lancet, vol. 352, no. 9140 , p. 1631, 1998.

[21] V. M. Hayes, R. M. W. Hofstra, C. H. C. M. Buys, H. Hollema, and A. G. J. Van Der Zee, "Homozygous arginine-72 in wild type p53 and risk of cervical cancer," Lancet, vol. 352, no. 9142, p. 1756, 1998.

[22] A. Nishikawa, T. Fujimoto, N. Akutagawa et al., "p53 polymorphism (codon-72) has no correlation with the development and the clinical features of cervical cancer," International Journal of Gynecological Cancer, vol. 10, no. 5, pp. 402-407, 2000.

[23] S. J. Klug, M. Ressing, J. Koenig et al., "TP53 codon 72 polymorphism and cervical cancer: a pooled analysis of individual data from 49 studies," The Lancet Oncology, vol. 10, no. 8, pp. 772-784, 2009.

[24] G. L. Bond, W. Hu, E. E. Bond et al., "A single nucleotide polymorphism in the MDM2 promoter attenuates the p53 tumor suppressor pathway and accelerates tumor formation in humans," Cell, vol. 119, no. 5, pp. 591-602, 2004.

[25] C. Menin, M. C. Scaini, G. L. De Salvo et al., "Association between MDM2-SNP309 and age at colorectal cancer diagnosis according to p53 mutation status," Journal of the National Cancer Institute, vol. 98, no. 4, pp. 285-288, 2006.

[26] N. Dharel, N. Kato, R. Muroyama et al., "MDM2 promoter SNP309 is associated with the risk of hepatocellular carcinoma in patients with chronic hepatitis C," Clinical Cancer Research, vol. 12, no. 16, pp. 4867-4871, 2006.

[27] S. S. Lum, H. W. Chua, H. Li et al., "MDM2 SNP309 G allele increases risk but the $\mathrm{T}$ allele is associated with earlier onset age of sporadic breast cancers in the Chinese population," Carcinogenesis, vol. 29, no. 4, pp. 754-761, 2008.

[28] V. Meissner Rde, R. N. Barbosa, J. V. Fernandes, T. M. Galvao, A. F. Galvao, and G. H. Oliveira, "No association between SNP309 promoter polymorphism in the MDM2 and cervical cancer in a study from northeastern Brazil," Cancer Detection and Prevention, vol. 31, no. 5, pp. 371-374, 2007.

[29] D. A. Arvanitis and D. A. Spandidos, "Deregulation of the G1/S phase transition in cancer and squamous intraepithelial lesions of the uterine cervix: a case control study," Oncology Reports, vol. 20, no. 4, pp. 751-760, 2008.

[30] H.-M. Lorenz, M. Herrmann, T. Winkler, U. Gaipl, and J. R. Kalden, "Role of apoptosis in autoimmunity," Apoptosis, vol. 5, no. 5, pp. 443-449, 2000.

[31] M. Zornig, A.-O. Hueber, W. Baum, and G. Evan, "Apoptosis regulators and their role in tumorigenesis," Biochimica et Biophysica Acta, vol. 1551, no. 2, pp. F1-F37, 2001.

[32] S. Nagata, "Apoptosis by death factor," Cell, vol. 88, no. 3, pp. 355-365, 1997.

[33] L. M. Butler, P. J. Hewett, W. J. Butler, and P. A. Cowled, "Down-regulation of Fas gene expression in colon cancer is not a result of allelic loss or gene rearrangement," British Journal of Cancer, vol. 77, no. 9, pp. 1454-1459, 1998.

[34] S. H. Lee, M. S. Shin, W. S. Park et al., "Alterations of Fas (APO-1/CD95) gene in transitional cell carcinomas of urinary bladder," Cancer Research, vol. 59, no. 13, pp. 3068-3072, 1999.

[35] T. Shimonishi, K. Isse, F. Shibata et al., "Up-regulation of fas ligand at early stages and down-regulation of Fas at progressed stages of intrahepatic cholangiocarcinoma reflect evasion from immune surveillance," Hepatology, vol. 32, no. 4, part 1, pp. 761-769, 2000.
[36] S. Kanemitsu, K. Ihara, A. Saifddin et al., "A functional polymorphism in fas (CD95/APO-1) gene promoter associated with systemic lupus erythematosus," Journal of Rheumatology, vol. 29, no. 6, pp. 1183-1188, 2002.

[37] Q. R. Huang, V. Danis, M. Lassere, J. Edmonds, and N. Manolios, "Evaluation of a new Apo-1/Fas promoter polymorphism in rheumatoid arthritis and systemic lupus erythematosus patients," Rheumatology, vol. 38, no. 7, pp. 645-651, 1999.

[38] Y. H. Lee, Y. R. Kim, J. D. Ji, J. Sohn, and G. G. Song, "Fas promoter -670 polymorphism is associated with development of anti-RNP antibodies in systemic lupus erythematosus," Journal of Rheumatology, vol. 28, no. 9, pp. 2008-2011, 2001.

[39] H.-C. Lai, H.-K. Sytwu, C.-A. Sun et al., "Single nucleotide polymorphism at Fas promoter is associated with cervical carcinogenesis," International Journal of Cancer, vol. 103, no. 2, pp. 221-225, 2003.

[40] M. T. Engelmark, K. Y. Renkema, and U. B. Gyllensten, "No evidence of the involvement of the Fas-670 promoter polymorphism in cervical cancer in situ," International Journal of Cancer, vol. 112, no. 6, pp. 1084-1085, 2004.

[41] A. Dybikowska, W. Sliwinski, J. Emerich, and A. J. Podhajska, "Evaluation of Fas gene promoter polymorphism in cervical cancer patients," International Journal of Molecular Medicine, vol. 14, no. 3, pp. 475-478, 2004.

[42] K. Sayama, S. Yonehara, Y. Watanabe, and Y. Miki, "Expression of Fas antigen on keratinocytes in vivo and induction of apoptosis in cultured keratinocytes," Journal of Investigative Dermatology, vol. 103, no. 3, pp. 330-334, 1994.

[43] D. N. Contreras, P. H. Krammer, R. K. Potkul et al., "Cervical cancer cells induce apoptosis of cytotoxic T lymphocytes," Journal of Immunotherapy, vol. 23, no. 1, pp. 67-74, 2000.

[44] H. Das, T. Koizumi, T. Sugimoto et al., "Quantitation of Fas and Fas ligand gene expression in human ovarian, cervical and endometrial carcinomas using real time quantitative RT-PCR," British Journal of Cancer, vol. 82, no. 10, pp. 1682-1688, 2000. 


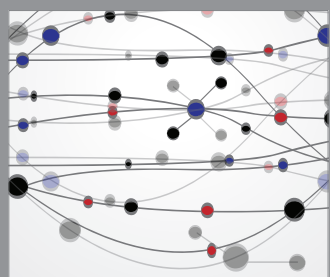

The Scientific World Journal
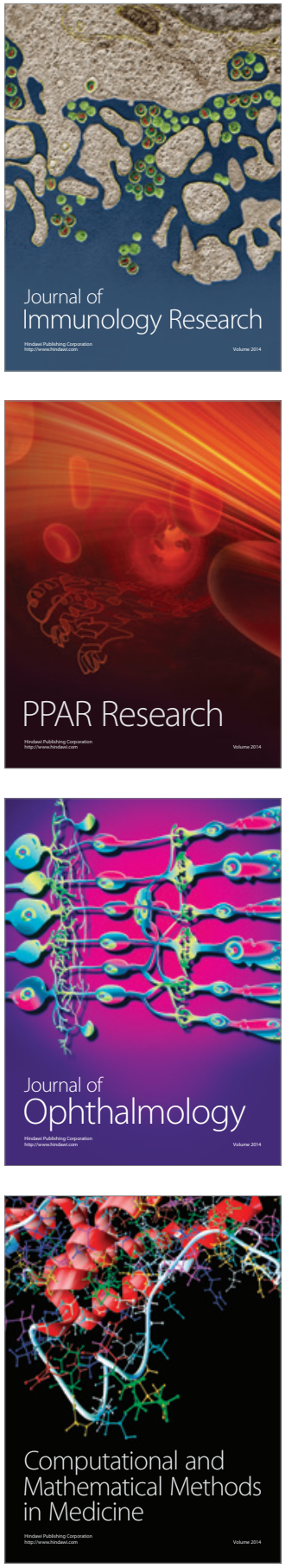

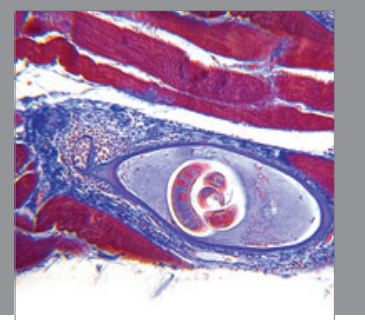

Gastroenterology

Research and Practice
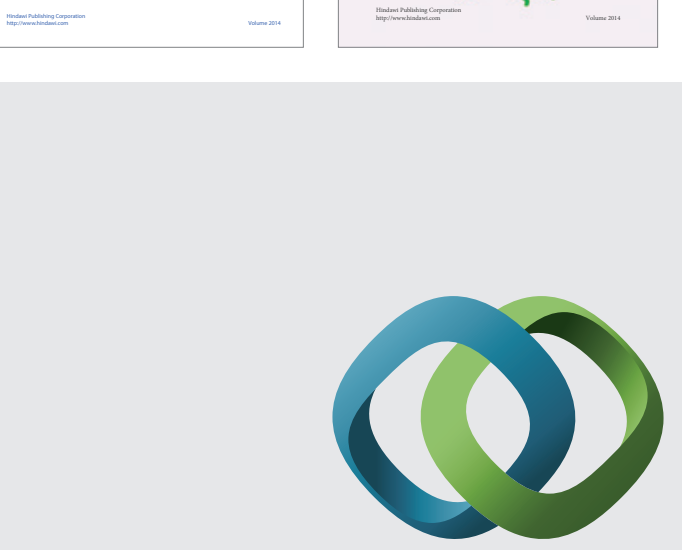

\section{Hindawi}

Submit your manuscripts at

http://www.hindawi.com
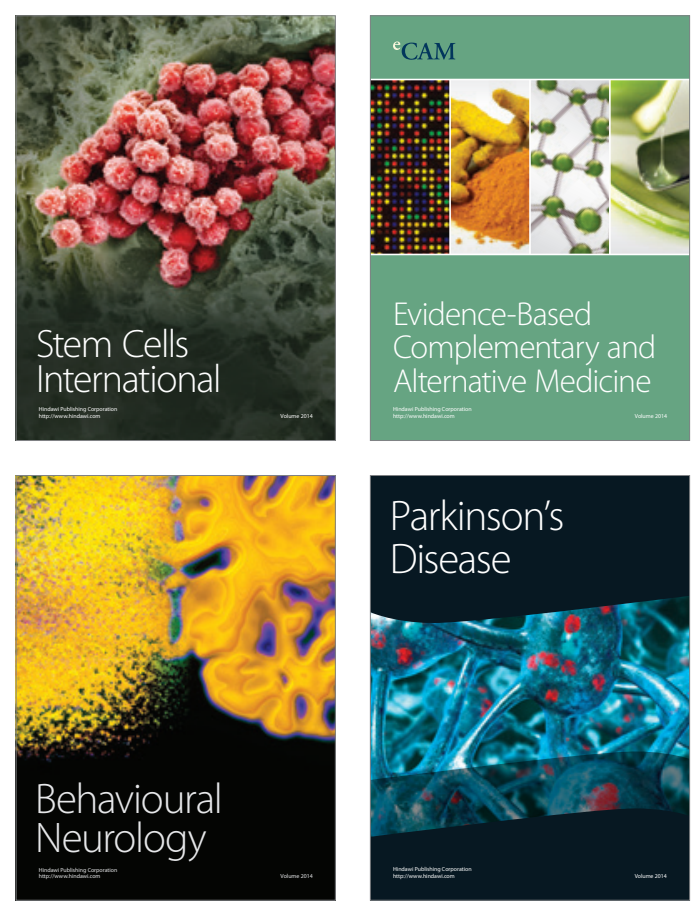

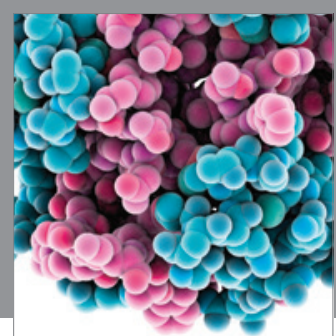

Journal of
Diabetes Research

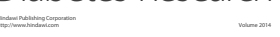

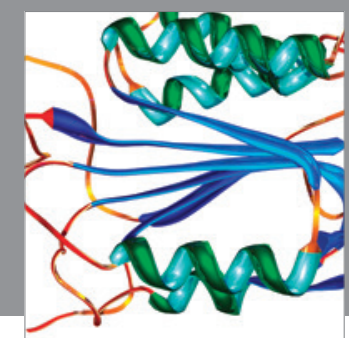

Disease Markers
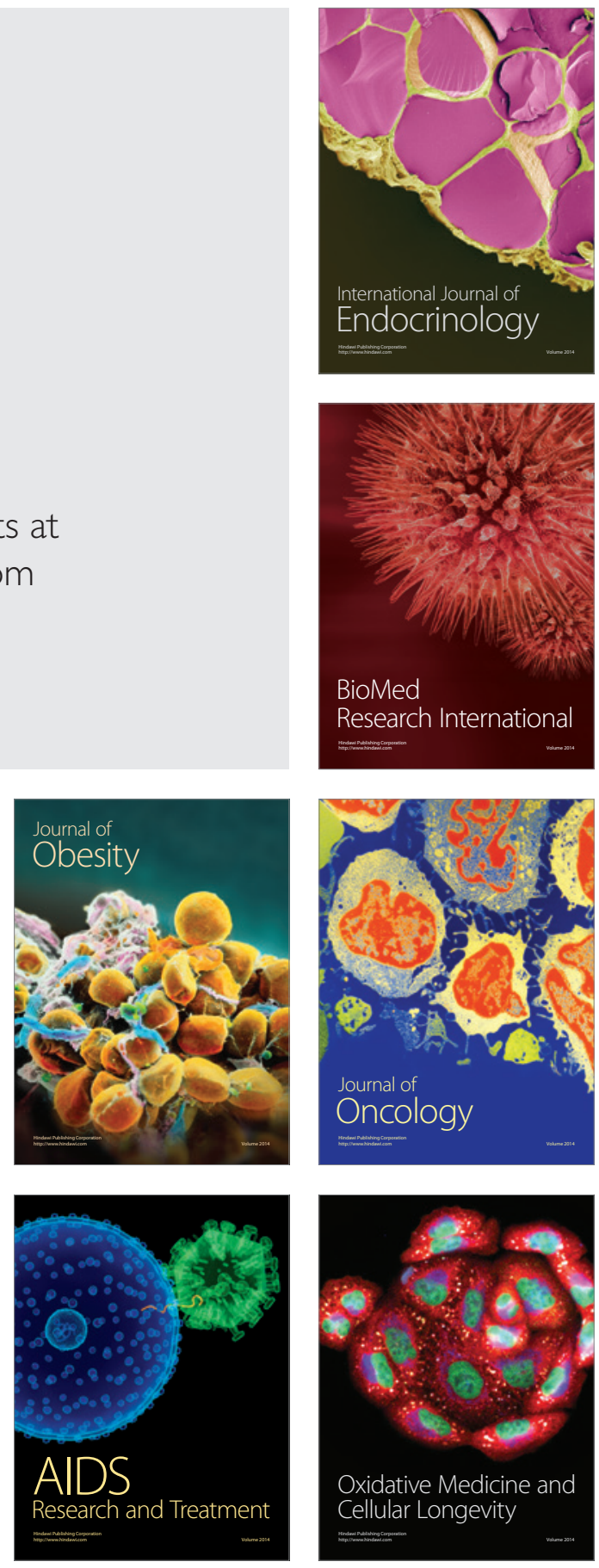